\title{
Transatlantica
}

Revue d'études américaines. American Studies Journal

2 | 2014

Aesthetics of Theory in the Modern Era and Beyond / Photographie documentaire

\section{Lionel Larré, Histoire de la nation cherokee}

Bordeaux, Presses Universitaires de Bordeaux, Collection Parcours

Universitaire, 2014

\section{Susanne Berthier-Foglar}

\section{(2) OpenEdition}

\section{Journals}

Édition électronique

URL : https://journals.openedition.org/transatlantica/7246

DOI : 10.4000/transatlantica.7246

ISSN : 1765-2766

Éditeur

Association française d'Etudes Américaines (AFEA)

Référence électronique

Susanne Berthier-Foglar, « Lionel Larré, Histoire de la nation cherokee », Transatlantica [En ligne], 2 |

2014, mis en ligne le 07 mars 2015, consulté le 01 février 2023. URL : http://journals.openedition.org/ transatlantica/7246; DOI : https://doi.org/10.4000/transatlantica.7246

Ce document a été généré automatiquement le 1 février 2023.

\section{(c) (i) $\odot$}

Creative Commons - Attribution - Pas d'Utilisation Commerciale - Pas de Modification 4.0 International - CC BY-NC-ND 4.0

https://creativecommons.org/licenses/by-nc-nd/4.0/ 


\title{
Lionel Larré, Histoire de la nation cherokee
}

\author{
Bordeaux, Presses Universitaires de Bordeaux, Collection Parcours \\ Universitaire, 2014
}

Susanne Berthier-Foglar

\section{RÉFÉRENCE}

LARRÉ, Lionel, Histoire de la nation cherokee, Bordeaux : Presses Universitaires de Bordeaux, Collection Parcours Universitaire, 2014, 285 pages, ISBN 978-2-86781-929-2, 14 euros.

1 Histoire de la nation cherokee est un ouvrage original qui traite du peuple cherokee depuis son apparition dans le sud-est des États-Unis jusqu'à l'époque contemporaine. L'originalité de cette Histoire vient du choix des documents, en version longue, qui accompagnent chaque chapitre et qui font partie des sources sur lesquelles se base sa rédaction. On y lit la voix des explorateurs en terre cherokee, de l'administration américaine, mais aussi du peuple cherokee lui-même. C'est en effet le premier peuple autochtone d'Amérique du Nord à avoir créé un système d'écriture de sa langue, le syllabaire mis au point par Sequoyah en 1821.

2 Le parti pris de Lionel Larré est d'expliquer pourquoi aujourd'hui, au $\mathrm{XXI}^{\mathrm{e}}$ siècle, il existe trois tribus cherokees officiellement reconnues par le gouvernement des ÉtatsUnis: la Nation Cherokee, en tant qu'entité politique, les Keetowah Unis et les Cherokees de l'Est. La " nation cherokee » du titre de l'ouvrage, sans majuscules, est le peuple cherokee, dans son intégralité, dont l'histoire est présentée ici, dans le contexte de la colonisation du continent américain par des nouveaux-venus d'Europe.

3 Lionel Larré choisit de donner la parole aux Cherokees tant que possible. Dans cette perspective, l'histoire des Cherokees commence par leur mythe de la création qui place le peuple au centre de l'univers et de la terre de ses ancêtres, en commençant par « premier homme » et "première femme ». À l'inverse, l'histoire du peuplement, telle 
que la racontent les Blancs, par une migration depuis la Sibérie, rabaisse les Cherokees au rang de peuple immigré comme les autres (15).

4 Sur les 13 chapitres de l'ouvrage, les 3 premiers sont centrés sur le monde cherokee tel qu'il a existé avant le contact avec l'Occident, ou du moins en marge d'un monde colonial qui n'avait pas encore empiété de façon trop radicale sur les terres cherokees. Les sources pour ces chapitres sont les écrits des explorateurs, produits à une époque où les contacts entre le peuple cherokee et l'Occident étaient sporadiques. Pour plus de réalisme historique, Lionel Larré fait appel à des sources contradictoires, ce qui rend compte de la difficulté de l'écriture de l'histoire mais aussi des différences dans l'appréciation d'un peuple qui ne correspond pas à la norme européenne. Ainsi, le lieutenant Timberlake voyait dans les Cherokees des primitifs occupés à faire des cérémonies conjuratoires inutiles (38), alors que James Adair, marchand éclairé parmi les Indiens, considérait leur savoir médical suffisamment avancé pour préférer un chirurgien cherokee à un Anglais pour le traitement d'une blessure ouverte, par balle ou par flèche (40).

Dans ces premiers chapitres sur la société cherokee non encore transformée par le contact avec l'homme blanc, Lionel Larré pose la question de la vision androcentrée des sources qu'il utilise et de la difficulté de mettre en évidence le rôle des femmes en politique et dans les faits de guerre (31). Un Européen des XVII et $\mathrm{xVIII}^{\mathrm{e}}$ siècles n'envisageait pas que les femmes puissent avoir ce type de rôle et les récits des voyageurs sont à décoder pour mettre en évidence la place de ces femmes honorées par le titre de ghigau (Bien-aimée) pour leur position dans la société cherokee. En outre, le partage des tâches rendait les hommes responsables de la chasse, ce qui semblait normal aux visiteurs européens, mais laissait l'agriculture aux femmes, ce qui faisait dire qu'il s'agissait forcément d'une activité facile (56). Lionel Larré précise qu'il n'y a pas de chronique de voyage de cette époque écrite par une femme.

Les chapitres 4 et 5 placent le monde cherokee dans un contexte de géopolitique coloniale où les factions tribales se rangent du côté de la France ou de l'Angleterre, et où les Cherokees sont finalement englobés dans le conflit américain de la guerre de Sept Ans (la French and Indian War pour les Américains). Lionel Larré rend compte de la complexité des alliances et de la difficulté pour les intéressés d'en comprendre tous les aboutissants.

7 À partir du chapitre 6, les Cherokees se trouvent face aux États-Unis et à la volonté d'expansion de leurs États et en particulier de la Géorgie. L'histoire est alors présentée sous l'angle de vue du bras de fer entre les États et le pouvoir fédéral. La Géorgie voulait se débarrasser des Cherokees, quel que soit leur niveau d'acculturation, et l'élection du président Andrew Jackson, issu de la culture de la frontière, allait faciliter le passage de la loi qui permettrait de repousser les Indiens sur la rive ouest du Mississippi.

8 Cette Histoire de la nation cherokee analyse de façon particulièrement poignante les processus de négociation des traités où la corruption des signataires et l'utilisation de l'alcool comme moyen d'infléchir leur volonté étaient des moyens couramment utilisés (114). Lorsque ces méthodes s'avérèrent insuffisantes, la Géorgie harcela les Cherokees, jusqu'à ce qu'ils partent, d'abord de façon volontaire, puis poussés par l'armée (206).

9 Cette dynamique de spoliation a été favorisée par la faiblesse du gouvernement central du peuple cherokee. Elle explique le factionnalisme qui a mené à la formation des trois tribus cherokees qui existent aujourd'hui : la «Nation Cherokee» en Oklahoma, qui représente la majorité, les Keetowah Unis, qui sont les descendants des premiers 
Cherokee délocalisés à l'ouest du Mississippi, et le Cherokees de l'Est, descendants de ceux qui ont échappé à la déportation en Caroline du Nord.

La destinée étasunienne de la tribu est aussi une histoire de son assimilation, de l'adoption de divers traits de la société sudiste, comme le modèle de la plantation, y compris l'esclavage. Par ailleurs, le peuple cherokee se métisse et Lionel Larré nous présente une vision de la citoyenneté cherokee pour laquelle ce métissage n'est qu'un élément secondaire, autant culturel que génétique $(223,253)$. Le rapprochement avec la société étatsunienne confronte les Cherokees à la théorie anglo-saxonne de la hiérarchie des races, une des raisons pour éloigner les Indiens des Blancs, raison qui était alors plus avouable que la soif de terres des Géorgiens (182).

D'ailleurs, pour illustrer la prétendue infériorité de la race indienne, on utilisa le stéréotype de l'Indien chasseur qui ne correspondait pas à la réalité cherokee du $\mathrm{XIX}^{\mathrm{e}}$ siècle. Ainsi, après la destruction des fermes des cherokees, l'administration américaine se proposait, non sans ironie, de leur apprendre l'agriculture.

12 L'Histoire de la nation cherokee est aussi une histoire de résistance. Un peu plus de vingt ans après la relocalisation forcée en Oklahoma, alors que le peuple cherokee avait reconstruit des fermes et des villes, la guerre de Sécession les força à prendre parti même si leur idée principale était de défendre le territoire cherokee (240). Après la guerre, le pays était dévasté, la population réduite de 21.000 à 14.000 personnes et au moment où les Cherokees se relevaient de cette destruction, les Étatsuniens arrivèrent en masse (251), provoquant la fin du Territoire Indien.

Tout au long de cette Histoire, on relève l'absence de parti pris. Certains missionnaires iront jusqu'à la prison pour défendre les Cherokees (135), d'autres philanthropes se disant «amis des Indiens" pousseront l'administration à mettre en place la loi de parcellisation des terres qui sera si néfaste à la cohésion tribale (254). Cette façon honnête d'écrire l'histoire est réaliste et elle rend le texte vivant.

Il faut aussi relever les qualités didactiques du texte. Il s'agit d'expliquer au lecteur francophone la véritable signification des termes anglais. Ainsi, le Blanc installé au milieu des Indiens a souvent la qualité de trader, ce qui signifie marchand plutôt que commerçant (51). Les premiers Cherokees à quitter leur pays pour s'installer à l'ouest du Mississippi sont les Old Settlers; settler se traduit par colon, mais il faut l'entendre au sens premier du terme, "celui qui s'implante sur une terre " (140). De même, il faut préciser pour le lecteur français que la guerre de Sécession s'appelle guerre civile pour les Américains, ou guerre entre les États. Autre problème lexical, doit-on parler de déportation des Indiens lorsqu'on parle de la loi qui en anglais s'appelle Indian Removal Act ? Lionel Larré demande à revoir cette traduction généralement acceptée car le terme déportation est réservé à la Shoah, mais il note que le terme déplacement est bien neutre (186). Ces réflexions sur les mots sont importantes. Il s'agit après tout de la collection « Parcours universitaire ». Toutefois, cette Histoire de la nation cherokee mérite une plus large diffusion que celle du public ciblé par la collection. 
INDEX

Thèmes : Recensions

\section{AUTEURS}

\section{SUSANNE BERTHIER-FOGLAR}

Université Grenoble Alpes

ILCEA4 (F-38040) 